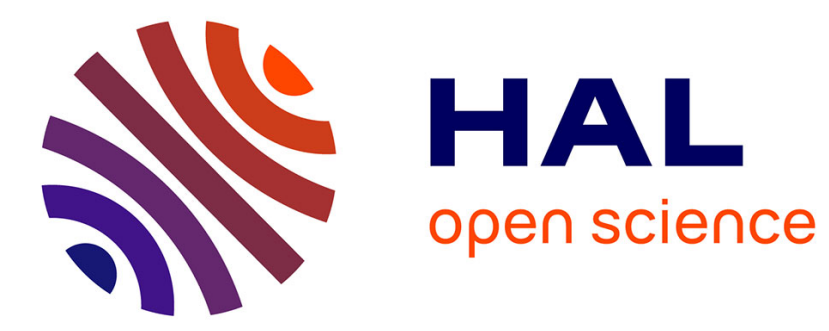

\title{
Nonlinear Tuned Mass Damper for self-excited oscillations
}

\author{
Vincenzo Gattulli, Di Fabio Fabio, Angelo Luongo
}

\section{To cite this version:}

Vincenzo Gattulli, Di Fabio Fabio, Angelo Luongo. Nonlinear Tuned Mass Damper for self-excited oscillations. Wind and Structures, 2004, 7 (4), pp.251-264. hal-00794927

\section{HAL Id: hal-00794927 \\ https://hal.science/hal-00794927}

Submitted on 26 Feb 2013

HAL is a multi-disciplinary open access archive for the deposit and dissemination of scientific research documents, whether they are published or not. The documents may come from teaching and research institutions in France or abroad, or from public or private research centers.
L'archive ouverte pluridisciplinaire HAL, est destinée au dépôt et à la diffusion de documents scientifiques de niveau recherche, publiés ou non, émanant des établissements d'enseignement et de recherche français ou étrangers, des laboratoires publics ou privés. 


\title{
Nonlinear Tuned Mass Damper for self-excited oscillations
}

\author{
Vincenzo Gattulli†, Franco Di Fabio ${ }^{\ddagger}$ and Angelo Luongo ${ }^{\ddagger \dagger}$ \\ Dipartimento di Ingegneria delle Strutture, delle Acque e del Terreno, Universita di L'Aquila, \\ 67040 Monteluco di Roio (L'Aquila), Italy
}

\begin{abstract}
The effects of a class of nonlinear Tuned Mass Dampers on the aeroelastic behavior of SDOF systems are investigated. Unlike classical linear TMDs, nonlinear constitutive laws of the internal damping acting between the primary oscillator and the TMD are considered, while the elastic properties are keept linear. The perturbative Multiple Scale Method is applied to derive a set of bifurcation equations in the amplitude and phase and a parametric analysis is performed to describe the postcritical scenario of the system. Both cubic- and van der Pol-type dampings are considered and the dependence of the limitcycle amplitudes on the system parameters is studied. These new results, compared with the previously obtained bifurcation scenario of a SDOF aeroelastic oscillator equipped with a linear TMD, show a detrimental effect on the maximum limit-cycle amplitude reduction of the nonlinear TMD. However, the analyses evidence that in the parameter region away from the perfect tuning condition the nonlinear connection can be used to tune the system with an enhancement of the limit-cycle amplitude reduction.
\end{abstract}

Keywords: structural control; wind engineering; nonlinear oscillations; bifurcations; post-critical behavior; perturbation methods.

\section{Introduction}

Protecting flexible structures against fatigue and unexpected instable behaviour due to aeolian induced oscillations constitutes a current technical challenge. Numerous different structures, going from traffic signal supports to overhead transmission lines, chimneys, towers and bridge decks, are prone to oscillate due to aeroelastic phenomena.

In many cases, a small mass is added to the structure aiming to transfer the aeolian energy entering in the primary system into a relative motion between the two sub-systems. The added mass, due to its inherent lightness, may oscillate with large amplitude causing a redistribution of both the elastic and dissipative energies.

Such protective device assumes different shapes and configurations depending on the structural system to be controlled and on how is connected to it. Most of the research for towers, buildings, chimneys and bridges have been devoted to the Tuned Mass Damper (TMD) used to prevent or reduce the magnitude of all different aeroelastic phenomena (e.g. Larsen 1993, Abdel-Rohman and Haskar 1996, Gattulli and Ghanem 1999, Strommen and Hjorth-Hansen 2001, Kwon 2002). Among several damper types, for overhead power transmission lines, the Stockbridge type damper has been

\footnotetext{
$\dagger$ Associate Professor $\ddagger$ Assistant Professor $\ddagger \dagger$ Professor
} 
one of the more applied (Hagedorn 1982, Markiewicz 1995). In many cases the connection that realizes the transfer of energy from the main system to the TMD is idealized as a linear spring and dashpot; however this simplification is in contrast with the fact that large relative displacement are expected, thus recent investigation are devoted to nonlinear TMD (Sauter and Hagedorn 2002, Lacarbonara and Vestroni 2002).

Investigations on the effects of an added mass have permitted to evaluate the optimal mechanical characteristics of the linear device which maximize the critical value at which the dynamic instability phenomenon occurs (Rowbottom 1981, Fujino and Abé 1993). However, in order to investigate system performance when the flow velocity exceeds the critical value, an analysis of the post-critical behavior is needed. Investigations on the system postcritical behavior has been performed by means of both numerical, analytical and experimental methods (Fujino, et al. 1985, Abdel-Rohman 1994). A first study of the system postcritical behavior as a 2DOF system has been presented by the authors in Gattulli, et al. (2001), with the aim to describe the postcritical scenario in the complete parameter-space. In this study, the primary system (PS) and the added mass (TMD) are assumed to posses a SDOF and to be linear, with the only source of nonlinearities arising from the flow-structure interaction. Using a perturbation method, simple and double Hopf bifurcations, occurring at different values of the parameters, have been analyzed. The effectiveness of TMDs has been shown to persist even in the postcritical range, since TMDs generally reduce the amplitude of oscillations in the supercritical case. However, the analysis developed, was only partial, since it was assumed that (a) a pair of conjugate eigenvalues of the Jacobian matrix is stable (simple Hopf) or (b) the two pairs are both critical but distinct (nonresonant double Hopf). In a second paper (Gattulli, et al. 2003) the same model has been considered and the postcritical behavior of the system analyzed for a Hopf bifurcation in the region of 1:1 resonance. The novel analysis leads to a second-order complex bifurcation equation in the amplitude of the unique critical mode. This has permitted to analyze the entire postcritical scenario in the bifurcation parameter space, evidencing the limits of validity of the concept of equivalent single DOF introduced in Fujino, et al. (1985), Abdel-Rohman (1994). An important conclusion of the past analysis is the following: if the control parameters are selected to maximize the critical wind velocity (optimal TMD), then the limit cycle amplitude for large velocities also reaches a minimum. Therefore the optimal TMD keeps its peculiarities even in the nonlinear range. However, since the control parameters are all determined by the required optimal conditions, no other parameters are available to try to further improve the system postcritical behaviour. With the aim to enhance the performance of the TMD, a suitable nonlinearity should be introduced in the system, able to reduce the wind effect after the critical condition. Since it is easy to show that a nonlinear cubic damping $\xi_{n} \dot{q}^{3}\left(\xi_{n}>0\right)$ added on a SDOF aeroelastic oscillator reduces the limit cycle amplitude, it is reasonable to investigate its effects, as internal force, also on a 2DOF system (i.e., the PS equipped with a TMD). The paper exploits the effectiveness of a nonlinear cubic damping in the TMDs on the bifurcation scenario of a simple aeroelastic oscillators comparing the new results with the results obtained for a linear TMD. The study evidences that the examined nonlinearity in general produces a mistuning effect together with an amplitude dependent damping. The mistuning effect destroyes the best behaviour of the optimal, perfectly tuned, linear TMD.

\section{Problem formulation}

An elastically supported bluff body connected with a small added mass and subject to a steady 


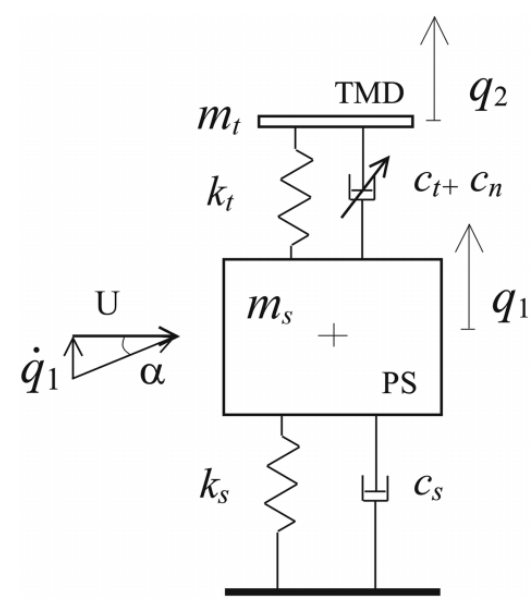

Fig. 1 Aeroelastic oscillator with tuned mass damper

flow is considered (Fig. 1). Both the bluff body primary system (PS) and the added mass (TMD) are assumed to posses a SDOF. The PS is supposed to be linear while the connection between the PS and the TMD is nonlinear, having the following constitutive relation:

$$
g\left(q_{1}, q_{2}, \dot{q}_{1}, \dot{q}_{2}\right)=m_{t} \omega_{t}^{2}\left(q_{1}-q_{2}\right)+2 m_{t} \omega_{t} \xi_{t}\left(\dot{q}_{1}-\dot{q}_{2}\right)+f\left(q_{1}, q_{2}, \dot{q}_{1}, \dot{q}_{2}\right)
$$

Eq. (1) accounts for linear elastic forces, damping forces and nonlinear forces. The aerodynamic forces acting on the TMD are assumed to be negligible in comparison with those acting on the PS. These forces are obtained using the quasi-static theory (Novak 1969), taking into account both drag and lift components and retaining the linear and cubic terms of their Taylor expansion.

By using nondimensional quantities and adopting a state-space representation the equations of motion for the system depicted in Fig. 1, read:

$$
\dot{\mathbf{x}}=\mathbf{L} \mathbf{x}+\mathbf{f}(\mathbf{x}, \mathbf{m})
$$

In Eq. (2)

$$
\mathbf{L}=\left[\begin{array}{cc}
\mathbf{0} & \mathbf{I} \\
-\mathbf{K} & -\mathbf{C}
\end{array}\right]
$$

is the system matrix with

$$
\mathbf{C}=\left[\begin{array}{cc}
2 \xi_{s}(1-v)+2 \mu \gamma \xi_{t} & -2 \mu \gamma \xi_{t} \\
-2 \gamma \xi_{t} & 2 \gamma \xi_{t}
\end{array}\right] \quad \mathbf{K}=\left[\begin{array}{cc}
1+\mu \gamma^{2} & -\mu \gamma^{2} \\
-\gamma^{2} & \gamma^{2}
\end{array}\right]
$$

the damping and stiffness matrices, respectively; $\mathbf{x}=\left\{q_{1}, q_{2}, \dot{q}_{1}, \dot{q}_{2}\right\}^{T}$ is the state space vector, with $q_{1}$ and $q_{2}$ the nondimensional cross-flow displacements of both PS and TMD, respectively; $\mathbf{m}=$ $\left\{\mu, \gamma, \xi_{t}, v\right\}$ is the vector of control parameters; $\mathbf{f}=\left\{f_{i}\right\}$ collects the nonlinear part of the vector field including aerodynamic and TMD nonlinear effects. The expression of the components $f_{i}$ are 
given in Appendix A for two selected cases of TMD nonlinear dampings, namely the cubic and van der Pol's dampings.

In Eq. (2) the following nondimensional variables have been introduced:

$$
\begin{gathered}
q_{1}=\frac{\hat{q}_{1}}{D} ; q_{2}=\frac{\hat{q}_{2}}{D} ; \mu=\frac{m_{t}}{m_{s}} ; \gamma=\frac{\omega_{t}}{\omega_{s}} ; v=\frac{U}{U_{u n c}} \\
U=\frac{\hat{U}}{\omega_{s} D} ; U_{u n c}=\frac{2 \xi_{s}}{\delta A_{1}} ; \delta=\frac{1}{2} \frac{\rho_{a} D^{2}}{m_{s}} ; t=\hat{t} \omega_{s}
\end{gathered}
$$

where $D$ is a typical dimension of the body, $m_{s}, m_{t}, \xi_{a}, \xi_{t}$ are masses and damping coefficients, $\omega_{s}$ and $\omega_{t}$ are the undamped frequencies of the two isolated bodies, $U$ is the uniform flow velocity, $U_{u n c}$ its critical value for the uncontrolled structure; $A_{i}$ are the aerodynamic coefficients, $\rho_{a}$ the air density and $t$ the time, the hat denoting dimensional quantities.

\section{Bifurcation analysis}

The equations of motion (2) admit the equilibrium position $\mathbf{x}=\mathbf{0}$. The position is stable or unstable depending on the values of the parameters $\mathbf{m}$-especially on the distinguished parameter $v$ which accounts for the flow velocity. The problem has been analysed in Rowbottom (1981), Fujino and Abé (1993), Fujino, et al. (1985), Abdel-Rohman (1994) and completely described in Gattulli, et al. (2001), where analytical expressions of nonresonant and resonant double Hopf manifolds are given.

A 3D representation of the critical manifolds for a fixed value of $\mu$ in the $\left(v, \xi_{t}, \gamma\right)$-space is shown in Fig. 2(a). In particular, In Fig. 2(b), different sections of the critical manifolds, varying $\gamma$ are depicted in the $\left(v, \xi_{t}\right)$-plane evidencing the existence of peculiar point $P_{0} \equiv\left(v_{0}, \xi_{t 0}, \gamma_{0}\right)$, at which the critical flow velocity is maximized and a 1:1 resonant double Hopf bifurcation occurs. These critical manifolds are evaluated for selected values of the mass ratio $\mu$ and the primary system damping ratio $\xi_{s}$. However, if these parameters are allowed to change, it is possibile to describe the locus of points $P_{0}$ in the $\left(v, \mu, \xi_{s}\right)$-space representing the 1:1 resonant double Hopf manifold. Different sections of the manifold are depicted in Fig. 3. They show that the augment of $\xi_{\text {s }}$ produces

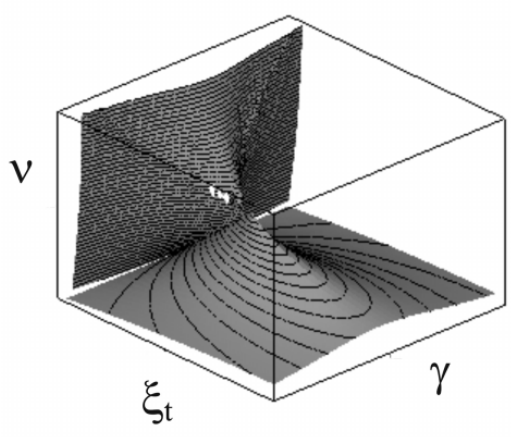

(a) 3D view

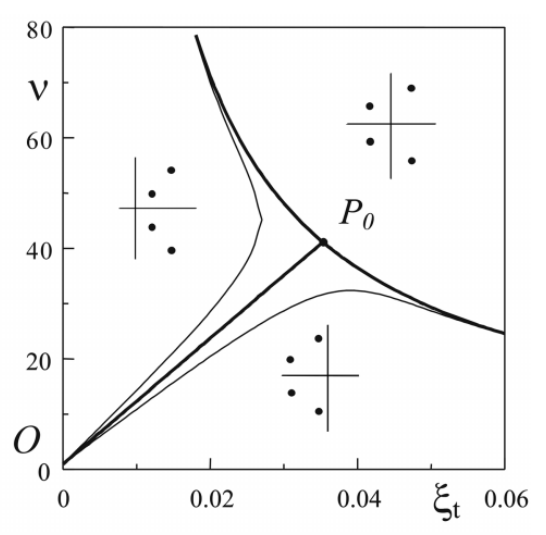

(b) Sections for different $\gamma$-values

Fig. 2 Critical manifolds in the space $\left(v, \xi_{t}, \gamma\right)$ for $\mu=0.005, \xi_{s}=8.8 e-3$ : (a) 3D view, (b) Sections for different $\gamma$-values 


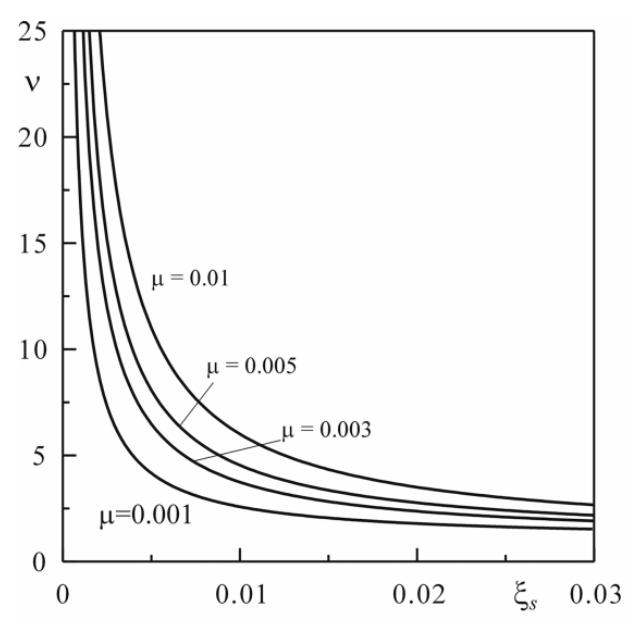

(a) at different $\mu$-values

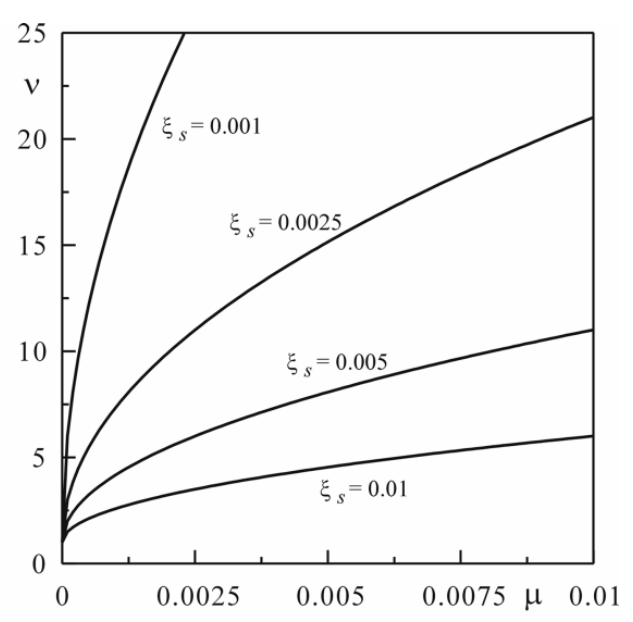

(b) at different $\xi_{\mathrm{s} \text {-values }}$

Fig. 3 Sections of the 1:1 double Hopf critical manifold in the $\left(\nu, \xi_{s}, \mu\right)$-space for $\gamma=\gamma_{0}$ and $\xi_{t}=\xi_{t 0}$ : (a) at different $\mu$-values, (b) at different $\xi_{s}$-values

smaller increment $v$ of the critical flow velocity in presence of a TMD with respect to the uncontrolled case (see Fig. 3(a)). Differently, larger values of $\mu$ produces the augment of the $v$ ratio (see Fig. 3(b)).

From this knowledge, the post-crtitical scenario is described starting from point $P_{0}$, through the procedure illustrated in Gattulli, et al. (2003) in which the Multiple Scale Method (MSM) is applied to perform the nonlinear analysis. Consequently, the deviations of the parameters from the bifurcation values $\left(v_{0}, \xi_{t 0}, \gamma_{0}\right)$ are assumed to be small, of order $\varepsilon^{2}$, with $\varepsilon$ a perturbation parameter, namely:

$$
v=v_{0}+\varepsilon^{2} v_{2}, \quad \gamma=\gamma_{0}+\varepsilon^{2} \gamma_{2}, \quad \xi_{t}=\xi_{t 0}+\varepsilon^{2} \xi_{t 2}
$$

where the incremental parameter $v_{2}, \xi_{t 2}$ and $\gamma_{2}$ represents, respectively, a distinguished parameter (positive for overcritical flow velocities) and the splitting parameters. Moreover, the state-space variables are expanded in series of integer powers of $\varepsilon$ as

$$
\mathbf{x}(t, \varepsilon)=\varepsilon \mathbf{x}_{1}+\varepsilon^{2} \mathbf{x}_{2}+\varepsilon^{3} \mathbf{x}_{3}+\varepsilon^{4} \mathbf{x}_{4}+O\left(\varepsilon^{5}\right)
$$

and several independent temporal scales $t_{k}=\varepsilon^{k} t(k=0,1, \ldots)$ are introduced, so that $d / d t=d_{0}+\varepsilon d_{1}$ $+\ldots$, with $d_{k}:=\partial / \partial t_{k}$. By substituting the previous equations in the Eqs. (2) and collecting terms with the same powers of $\varepsilon$, the following perturbation equations are drawn up to the $\varepsilon^{4}$-order:

$$
\begin{aligned}
& \left(\mathbf{L}_{0}-\mathrm{d}_{0}\right) \mathbf{x}_{1}=0 \\
& \left(\mathbf{L}_{0}-\mathrm{d}_{0}\right) \mathbf{x}_{2}=\mathrm{d}_{1} \mathbf{x}_{1} \\
& \left(\mathbf{L}_{0}-\mathrm{d}_{0}\right) \mathbf{x}_{3}=\mathrm{d}_{1} \mathbf{x}_{2}+\left(\mathrm{d}_{2}-\mathbf{L}_{2}\right) \mathbf{x}_{1}-\frac{1}{6} \mathbf{f}_{\mathbf{x x x}}^{0} \mathbf{x}_{1}^{3} \\
& \left(\mathbf{L}_{0}-\mathrm{d}_{0}\right) \mathbf{x}_{4}=\mathrm{d}_{1} \mathbf{x}_{3}+\left(\mathrm{d}_{2}-\mathbf{L}_{2}\right) \mathbf{x}_{2}-\frac{1}{2} \mathbf{f}_{\mathbf{x x x}}^{0} \mathbf{x}_{1}^{2} \mathbf{x}_{2}
\end{aligned}
$$

In Eqs. (8) $\mathbf{L}_{2}$ is the second-order part of the $\varepsilon$-expansion of $\mathbf{L}$ around $P_{0}$ (i.e., $\mathbf{L}=\mathbf{L}_{0}+\varepsilon^{2} \mathbf{L}_{2}$ ), and 
$\mathbf{f}_{\mathbf{x x x}}^{0}$ is the third derivative of the nonlinear part of the vector field at $P_{0}$, evaluated for both the cubic and van der Pol dampings operating on the nonlinear fields reported in Appendix-I which includes the aerodynamic nonlinearities.

It should be noted that, in deriving $\mathbf{f}_{\mathbf{x x x}}^{0}$, the actual value $U$ of the flow velocity has been considered, instead of the bifurcation value $U_{0}$ (Novak 1969). Although this procedure is inconsistent, numerical results have shown that it improves the accuracy of the solution for $U \gg U_{0}$ (Gattulli, et al. 2001).

Imposing the solvability conditions and combining them in a unique equation through a consistent reconstitution procedure, the evolutive equation for the post-critical complex amplitude $A$ follows (see also Gattulli, et al. 2003),

$$
\frac{\mathrm{d}^{2} A}{\mathrm{~d} t^{2}}=C_{1} A+C_{2} \frac{\mathrm{d} A}{\mathrm{~d} t}+C_{3} A^{2} \bar{A}+C_{4} A \bar{A} \frac{\mathrm{d} A}{\mathrm{~d} t}+C_{5} A^{2} \frac{\mathrm{d} \bar{A}}{\mathrm{~d} t}
$$

In Eq. (9) the parameter $\varepsilon$ has been adsorbed according with $\varepsilon A \rightarrow A, \varepsilon \mathrm{d} / \mathrm{d} t \rightarrow \mathrm{d} / \mathrm{d} t$ and the coefficients $C_{i}$ are reported in the Appendix B. Expressing the amplitudes in polar form $A=1 / 2 a\left(t_{2}\right) e^{i \theta\left(t_{2}\right)}$ and separating the real and imaginary parts of Eq. (9), four differential equations of the first order in the real variables $(a, \theta, r, s)$ follow

$$
\begin{aligned}
& \dot{a}=r \\
& \dot{r}=R_{1} a+a s^{2}+\frac{1}{4} R_{3} a^{3}+R_{2} r-I_{2} a s+\frac{1}{4}\left(R_{4}+R_{5}\right) a^{2} r+\frac{1}{4}\left(I_{5}-I_{4}\right) a^{3} s \\
& a \dot{s}=I_{1} a-2 r s+\frac{1}{4} I_{3} a^{3}+R_{2} a s+I_{2} r+\frac{1}{4}\left(I_{4}+I_{5}\right) a^{2} r+\frac{1}{4}\left(R_{4}-R_{5}\right) a^{3} s \\
& \dot{\theta}=s
\end{aligned}
$$

In Eq. (10) $a$ is the modal amplitude of the motion, $\theta$ the phase, and $s$ the frequency correction, so that the steady-state motion at the leading order, reads:

$$
\mathbf{x} \simeq \varepsilon \mathbf{x}_{1}+\varepsilon^{2} \mathbf{x}_{2}=1 / 2 a\left(\mathbf{u}_{1}+i s \mathbf{u}_{2}\right) e^{\left(i \Omega t+\theta_{0}\right)}+c . c .
$$

where $\mathbf{u}_{1}$ and $\mathbf{u}_{2}$ are, respectively, the proper and the generalized eigenvectors of $\mathbf{L}$ at the critical point $P_{0}$, and $\Omega=\omega+s$ is the nonlinear frequency; moreover the coefficients $R_{i}$ and $I_{i}$ are dependent on the linear and nonlinear parameters. In Eqs. (10a-10c), the unknown variables $(a, r, s)$, are in number equal to the codimension of the problem; the variables describe the post-critical behavior of the system (2) in the region of a 1:1 resonant double Hopf bifurcation. Eq. (10d), decoupled from the previous equations, describes the evolution of the phase $\theta$.

The steady-state solutions of (10) are obtained by zeroing the right-hand side terms of (10a-10c). Since a cubic equation in $a^{2}$ can be drawn, a discussion of the solutions of the cubic equation is necessary to display the complete scenario. Indeed, depending on the control parameters values, different solutions may occur such as a three real non-trivial solutions $(a, s)$, a combination of one real $(a, s)$ and two purely imaginary solutions $\left(|a| e^{ \pm i \pi / 2}, s\right)$ or one real and a pair of complex conjugate solutions $\left(|a| e^{ \pm i \alpha},|s| e^{ \pm i \pi / 2}\right)$. The real solutions represent periodic motion (limit cycles) of 
the system (2) with constant amplitude $a$ and frequency $\Omega=\omega+s$. The solutions with purely imaginary amplitude $a$ and real frequency correction $s$ still represent periodic motion (limit cycles) with a phase shift of $\pi / 2$, while the complex conjugate solutions represent respectively a diverging to infinity or converging to zero motion with exponentially varying amplitude $a(t)=|a| e^{ \pm I m(s) t}$.

\section{Effects of nonlinear TMDs on the bifurcation scenario}

The influence of the main system parameter on the post-critical behavior have been carried out to analyze the effects of the nonlinear TMD on the postcritical dynamics of the PS. In particular, investigation on the effects of two different nonlinear damping laws acting between the two subsystem has been considered, namely cubic and a van der Pol's damping. The analysis has been carried out on the basis of the illustrated analytical solutions as well as direct time-integration of the equations of motion. In both the studied cases, the nonlinear TMD solutions are compared with that relevant to the linear TMD, already obtained in Gattulli, et al. (2003).

\subsection{Cubic damping}

The influence of the main system parameter on the post-critical behavior is here presented in the case of nonlinear (NL) TMD with cubic damping. Steady solutions of the system are compared with linear (L) solutions. The comparison highlights the influence of the nonlinear parameter. In particular the qualitative change in the postcritical behavior depends only by the nonlinear damping while linear parameters such as $\mu$ and $\xi_{s}$ influence the behavior quantitatively. However, different TMDs has been analyzed in order to quantify these differences.

Selecting a TMD with optimum linear parameter, hence belonging to 1:1 resonant manifold, with values $\mu=0.005$ and $\xi_{s}=8.8 e-3$, the splitting parameters $\left(\xi_{t 0}, \gamma_{0}\right)$ are conseguently evaluated while the nonlinear aerodynamic coefficients depends on the symmetric cross-section shape (in particular
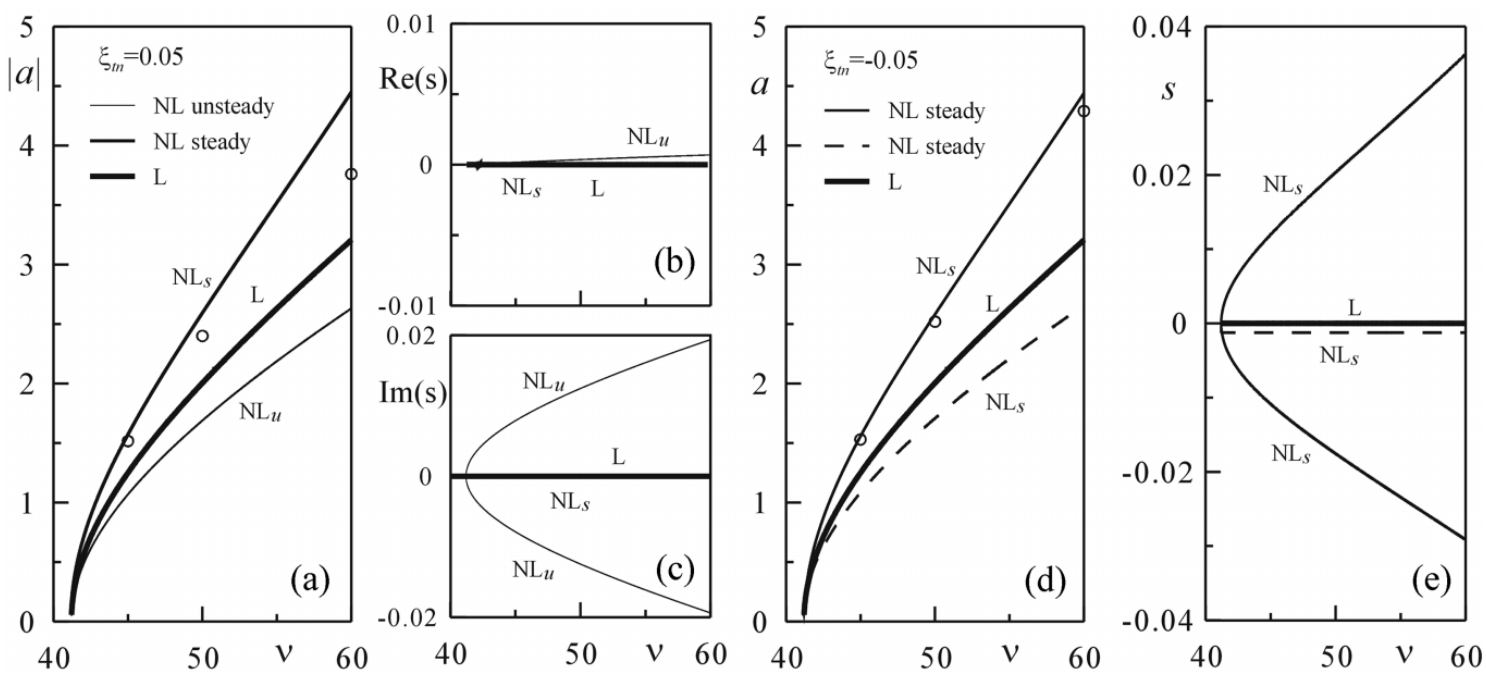

Fig. 4 Effects of cubic damping $\xi_{t n}$ on the galloping amplitudes $a$ and frequency correction $s$ at the optimal L-TMD (stable solid, unstable dotted lines): (a) (b) (c) $\xi_{t n}>0$, (d) (e) $\xi_{t n}<0$. 
for the selected square cross-section $A_{1}=4.87, A_{3}=-421$ ); therefore the limit cycle amplitude depends only on the bifurcation parameter $v$ (the flow velocity) as depicted on Fig. 4. In particular, the system posses three coincident real non-trivial solutions, $a=a(v), s=0$, corresponding to a unique optimal limit cycle at the lowest amplitude obtainable by a linear TMD. Perturbing such conditions through the addition of an internal nonlinear damping entails destroying this coalescence. In particular, if a positive $\xi_{\text {tn }}$ is considered, the three coincident real solutions are transformed in one real and a pair of complex conjugate solutions. Unfortunately, the real solution has an amplitude higher than the one of the linear case, as shown in Fig. 4(a). In the same figure a lower amplitude, associated with the complex conjugate solutions, appears associated to a divergent or convergent motion with constant amplitude. These three distinct solutions describe completely the possible system motion, depending on the initial conditions. For negative $\xi_{t n}$ three different real solutions are admitted (Fig. 4(d) and 4(e)). A pair of these are stable, having the same amplitude $a$, still higher than in the linear case, and opposite frequency corrections $s$. The third one is instead unstable, with lower amplitude of the linear case, and almost zero frequency correction. The previously described solutions are compared with that obtained through a direct time-integration of the system Eq. (2) with suitable initial conditions. The modal amplitude of the steady-state solutions for different flow velocities are marked by dots in Fig. 4 .

Moving to the comparison between linear and nonlinear TMD performance away from the optimal condition, in the region $\xi_{t}<\xi_{t 0}$ and $\gamma=\gamma_{0}$, where double Hopf bifurcation occurs, (Gattulli, et al. 2003), the scenario changes (Fig. 5). Indeed, the cubic equation in $a^{2}$ for the linear case admits a real unstable solution $a=a(v), s=0$, and a pair of stable solutions with same amplitude and opposite frequency corrections. The presence of a positive $\xi_{t n}$, in this case, enhances the performance of the linear TMD. Indeed it permits the system to reach the perfect tuning condition that constitutes the optimal solution. Looking at Fig. 5(b), the detuning caused by the linear parameter $\xi_{t} \neq \xi_{t 0}$ is completely recovered by the nonlinear TMD increasing the flow velocity. This path coincides with a decreasing of the two stable solutions with same amplitude and opposite frequency correction. At the same time, the unstable solution is augmented in amplitude by $\xi_{\text {tn }}$, such that, at certain level of flow velocity it crosses the stable solutions in a point where the three
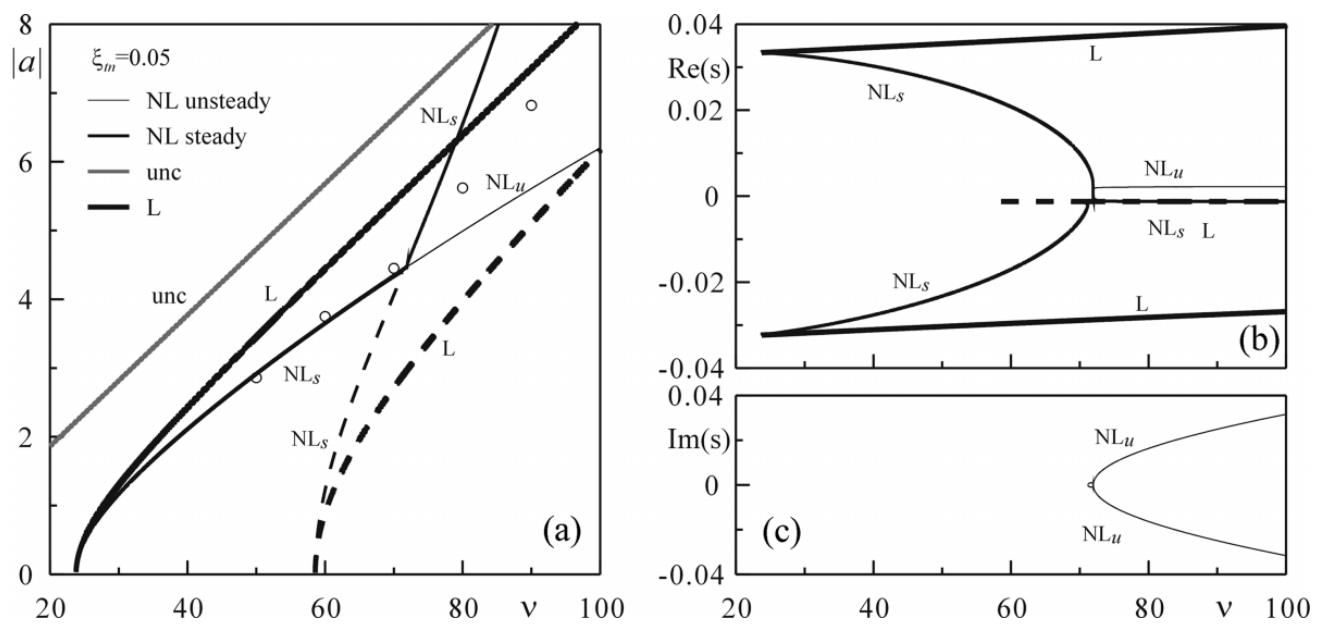

Fig. 5 Effects of TMD cubic damping $\xi_{t n}$ on the galloping amplitudes away from the linear optimal TMD $\left(\mu=0.005, \xi_{s}=8.8 e-3\right)$ (stable solid, unstable dotted lines): (a) amplitudes, (b), (c) frequency corrections vs flow velocity 

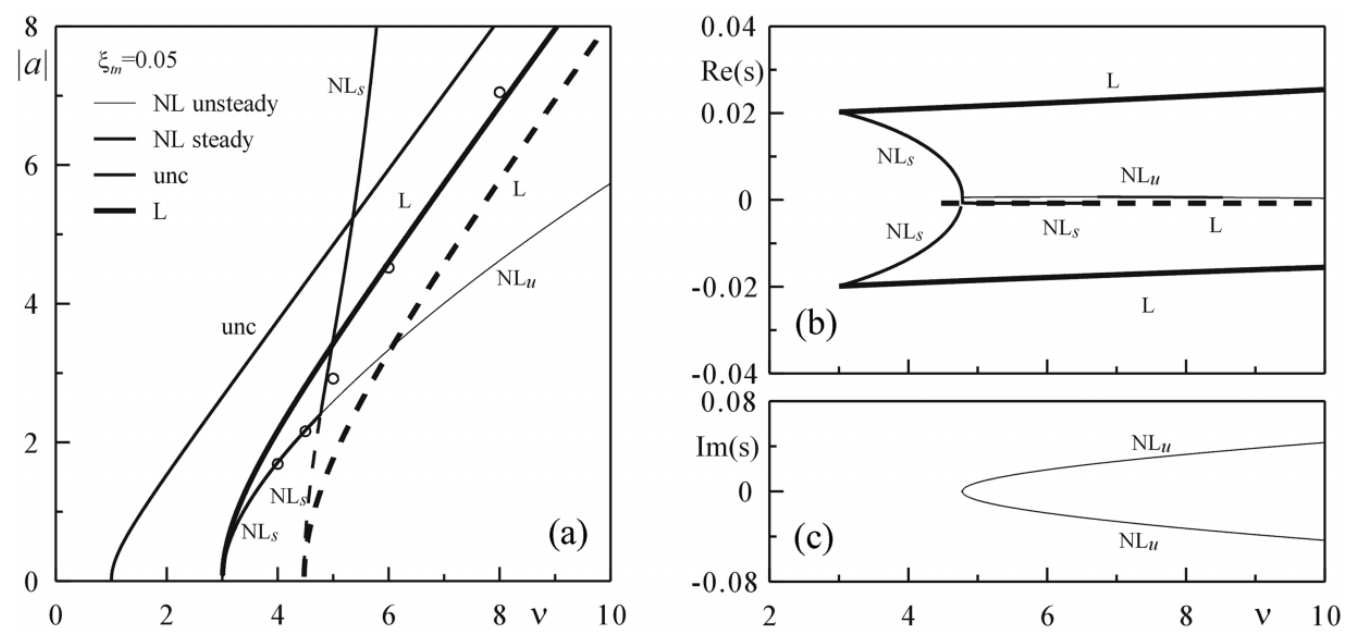

Fig. 6 Effects of TMD cubic damping $\xi_{\text {tn }}$ on the galloping amplitudes away from the linear optimal TMD for a different system $\left(\mu=0.003, \xi_{s}=0.01\right)$ (stable solid, unstable dotted lines): (a) amplitudes, (b),(c) frequency corrections vs flow velocity

solutions coincide, having zero frequency correction and the same amplitude. The curve crossing produces a modification of the stability of the solution, such that the unstable solutions become stable and the two real stable solutions become complex conjugates, representing converging and diverging motions as previously explained.

The same qualitative scenario is encountered also for a TMD, still belonging to the resonant manifold, but with different $\mu(\mu=0.003)$ acting on a system with greater inherent damping $\left(\xi_{s}=0.01\right)$ (Fig. 6). The main differences with the previous case is that the critical flow velocity ratio is much smaller, accordingly with the results presented in Fig. 3. Therefore, the post-critical behavior is more likely to occur. In this case a perfect agreement with results of direct time integration has been found up to the bifurcation point (dots in Fig. 6(a)), while for high wind speed the accuracy is loss.

Nevertheless, an interesting question regards the amplitude level reached by the previously perfectly tuned solutions obtained through the nonlinear damping, compared with the one of the optimal linear case. Fig. 7(a) compares the amplitudes obtained by linear TMDs with different $\xi_{t}$ (bold lines) with the same ones enhanced by a nonlinear damping (fixed at $\xi_{t n}=0.05$ ). Following the curves for a given flow velocity $(v=40)$, it is clear that the addition of nonlinear damping succeeds in reducing the limit-cycle amplitude in the region of low linear damping $\left(\xi_{t} \neq \xi_{t 0}\right)$; in contrast when the linear damping is greater than the optimal value, the addition of cubic nonlinear damping is detrimental. The increasing of the flow velocity $(v=60 ; v=80)$ evidences that the locus of the minimum amplitude value belonging to the straight line $C$ for the linear TMD (see also Gattulli 2003) is distorted by the cubic damping in the curve $C_{n}$, along which a new minimum value is reached.

On this new scenario, the interest has been finally focused on the existence of the absolute minimum amplitude value obtainable for linear and nonlinear TMDs for a given flow velocity. The search has been conducted varying the $\left(\xi_{t}, \xi_{t n}\right)$-pair for the nonlinear TMD and comparing its performance with the "optimal" linear TMD. The results are presented in Fig. 7(b) where the stable 


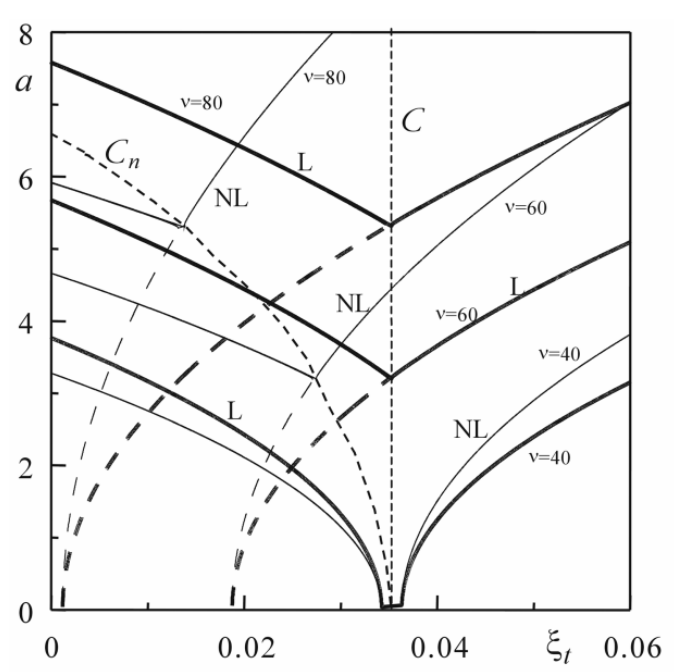

(a) $\xi_{\mathrm{t}}$

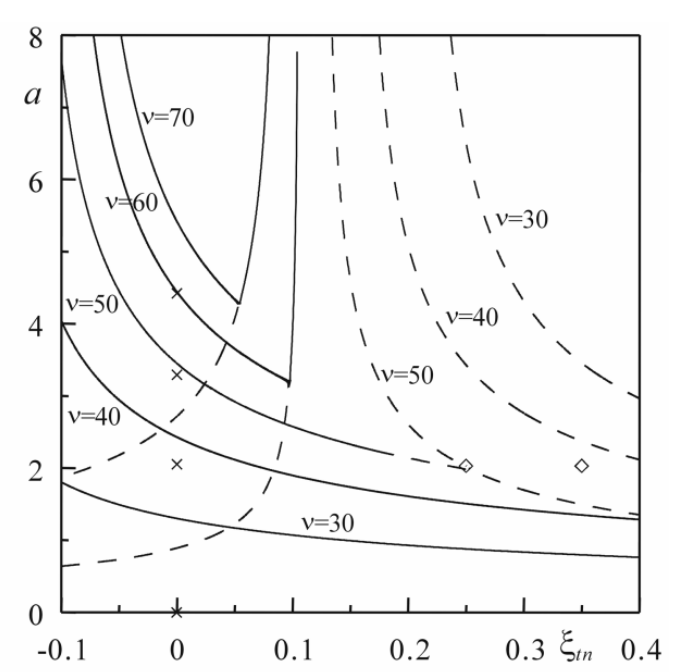

(b) $\xi_{\text {tn }}$

Fig. 7 Limit cycle amplitudes for linear (L) and nonlinear (NL) TMD's vs damping coefficients (stable solid, unstable dotted lines): (a) $\xi_{t}$, (b) $\xi_{t n}$

limit-cycle amplitude are described as function of the cubic nonlinear damping in the $\left(a, \xi_{t n}\right)$-plane for a given $\xi_{t}\left(\xi_{t}=0.02\right)$. For selected flow velocities $(v=30 ; v=40)$ the addition of nonlinear damping cannot produce a reduction with respect the optimum linear TMD because at this level of flow the system is not galloping (the linear TMD reference amplitude, depicted as a cross $(\times)$, belongs to the horizontal axis). Increasing the flow velocity $v$, both the compared systems behave in the postcritical range; augmenting the cubic damping to the linear one $\left(\xi_{t}=0.02<\xi_{t 0}\right)$ produces a decrease of the amplitude up to a certain value where the solution becomes unstable. In these cases, comparing the results for linear and nonlinear TMD permits to evidence some special situations (depicted as diamonds in Fig. 7(b)) where the amplitude level is lower than the one obtained in the optimal linear case at the same flow velocity. For example, for a flow velocity of $v=50$, the limitcycle amplitude of the optimal linear TMD is $a=2.061$ while the nonlinear TMD $\left(\xi_{t}, \xi_{t n}\right)=(0.02$, 0.25 ) has an amplitude of $a=2.020$ with a reduction of $2.04 \%$.

\subsection{Van der Pol's damping}

The effects of the cubic damping on the limit-cycle amplitude have shown that in a very limited number of cases is possible to obtain a reduction with respect of the "optimal" linear TMD. This is mainly due to the particular effects that this type of nonlinearities produces on the bifurcation diagram presented in Fig. 4(a) where the lower amplitudes are associated to unsteady classes of motion. Consequently, a second investigation has been conducted on a different internal nonlinear damping, namely a van der Pol's nonlinear constitutive relation, as reported in Appendix I. The new coefficients of the steady-state solutions of (10) have been evaluated for the new internal nonlinear damping and the steady-state amplitudes have obtained as in the previous case. The nonlinear TMD solutions (NL) are again, directly compared with the linear TMD ones (L). The scenario obtained by the addition of the cubic damping is almost unchanged by the use of van der Pol's damping. In 

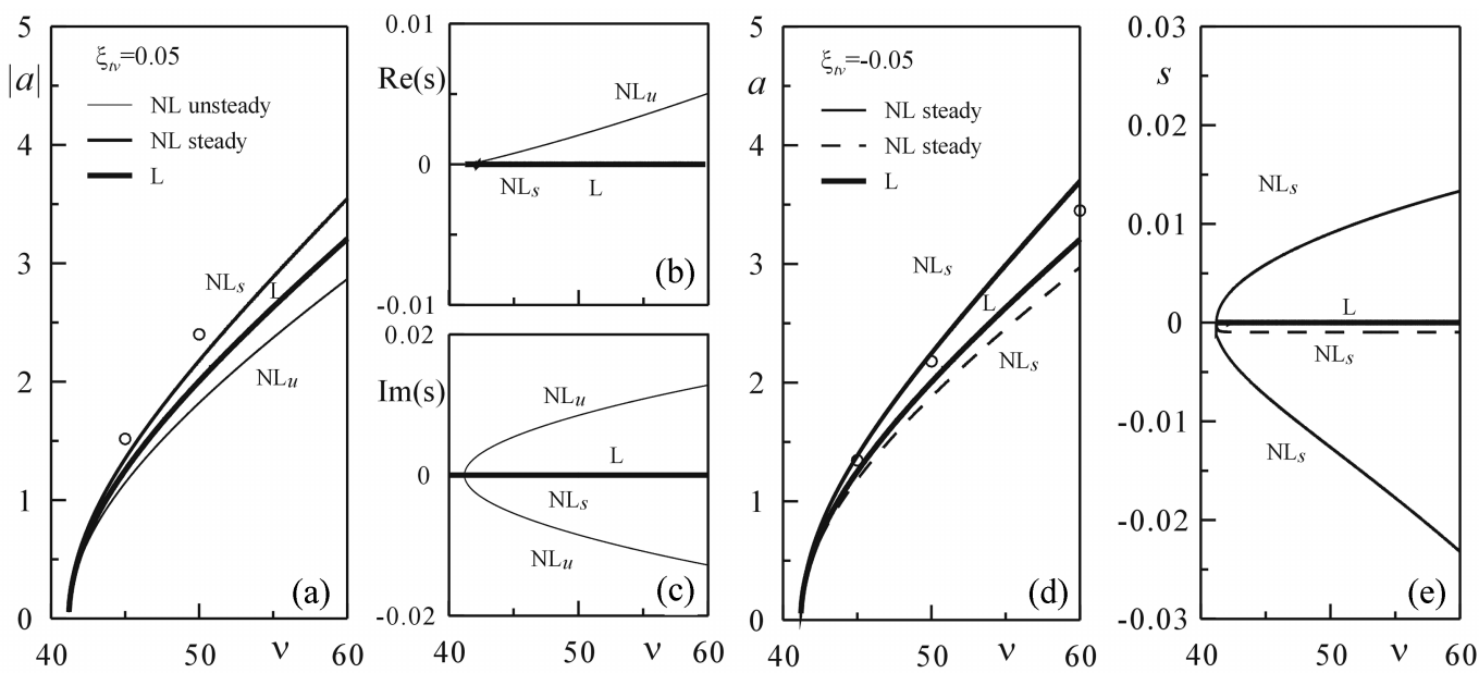

Fig. 8 Effects of van der Pol's damping $\xi_{t v}$ on the galloping amplitudes $a$ and frequency correction $s$ at the optimal L-TMD (stable solid, unstable dotted lines): (a) (b) (c) $\xi_{t v}>0$, (d), (e) $\xi_{t v}<0$
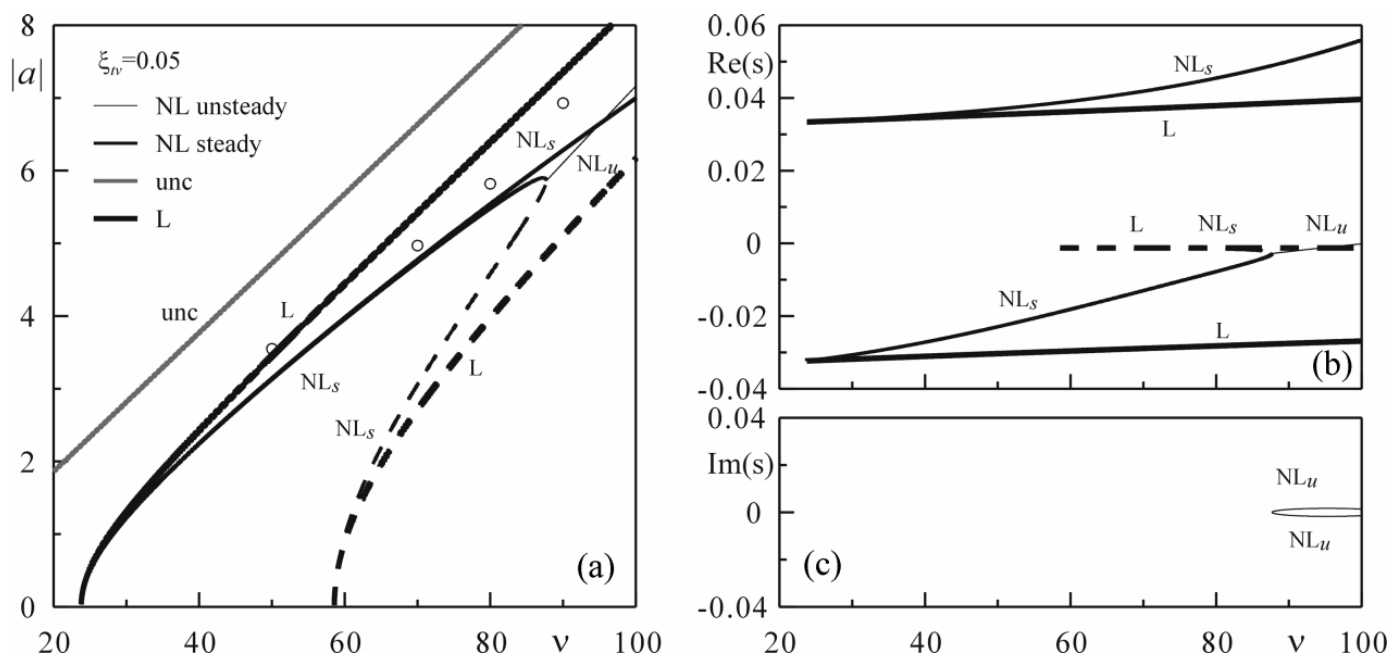

Fig. 9 Effects of van der Pol's damping $\xi_{t v}$ on the galloping amplitudes away from the linear optimal TMD (stable solid, unstable dotted lines): (a) amplitudes (b), (c) frequency corrections vs flow velocity

particular, Fig. 6 compares the unique "optimal" limit cycle of the linear TMD with these new solutions obtained for a different nonlinear damping. Again, the lower amplitudes correspond to unsteady solutions for $\xi_{t v}>0$ (Fig. 8(a)) and to unstable solutions for $\xi_{t v}<0$ (Fig. 8(d)). The main differences with respect to the previous case are in the frequency corrections $s$ (see Fig. 8(b),(c),(e)). Far from the optimal condition the type of nonlinearity influences more strongly the postcritical behavior. Indeed, the path of two coincident solutions at lower amplitude presented in Fig. 5(a), that bifurcates for a given flow velocity, change in a smoother behavior. Fig. 9(a) shows the paths of the two stable solutions that behave similarly up to a certain flow velocity, after that they follow 
different patterns. The first one becomes unstable after a limiting point and the second one grows, keeping itself stable for increasing flow velocities. The modification with respect to the cubic damping case appears more evident if the frequency correction $s$ in Fig. 9(b) are analyzed. It emerges that the difference in the behavior of the two stable solution is caused by a strong difference of the $s$-values of the steady-state solutions.

\section{Conclusions}

The effects of internal cubic damping on the postcritical behavior of an aeroelastic SDOF oscillator equipped with a Tuned Mass Damper have been analysed and the results compared with the more common linear case. A perturbative analysis has been performed by using the MSM around a double Hopf bifurcation in 1:1 resonance. This approach permits to span completely the entire post-critical scenario both for the linear and the nonlinear TMD cases. It has been found that the nonlinear internal damping is uneffective in the reduction of the limit cycle amplitude if the starting linear system is the optimal perfectly tuned one. Neverthless, if the comparisons are conducted in a region sufficiently far from the optimal linear case, a better nonlinear TMD exists with respect to the obtainable limit cycle amplitude, although this is triggered at lower flow velocity. The study posses a technical interest because the linear optimal region is generally very small and the optimality condition can been easily lost for a small variation in the linear parameter of both the structure and the TMD. Further investigation are needed for different nonlinear coupling between the PS and the TMD.

\section{Acknowledgements}

This work was partially supported by Italian Ministry of Education and Research (MIUR) under the project titled: Dynamic behavior of structures: theory and experiments (COFIN 01-02).

\section{References}

Abdel-Rohman, M. (1994), "Design of tuned mass dampers for suppression of galloping in tall prismatic structures", J. Sound Vib., 171, 289-299.

Abdel-Rohman, M. and Askar, H. (1996), "Control by passive TMD of wind-induced nonlinear vibrations in cable stayed bridges", J. Vib. Contr., 2, 251-267.

Fujino, Y. and Abé, M. (1993), "Design formulas for tuned mass dampers based on a perturbation technique", Earth. Eng. Struct. Dyn. 22, 833-854.

Fujino, Y., Warnitchai, P. and Ito, M. (1985), "Suppression of galloping of bridge tower using tuned mass damper", J. Fac. of Eng., Univ. of Tokyo, 38, 49-73.

Gattulli, V. and Ghanem, R. (1999), "Adaptive control of flow-induced oscillations including vortex effects", Int. J. Non-Linear Mech., 34, 853-868.

Gattulli, V., Di Fabio, F. and Luongo, A. (2001), "Simple and double Hopf bifurcations in aeroelastic oscillators with tuned mass dampers", J. Franklin Inst., 338, 187-201.

Gattulli, V., Di Fabio, F. and Luongo, A. (2003), "One to one resonant double hopf bifurcation in aeroelastic oscillators with tuned mass dampers", J. Sound Vib., 262, 201-217.

Hagedorn, P. (1982), "On the computation of damped wind-excited vibrations of overhead trasmission lines". $J$. Sound Vib., 83, 253-271.

Kwon, S. (2002), "Control of flutter of suspension bridge deck using TMD”, Wind Struct., An Int. J., 5(6), 563567. 
Lacarbonara, W. and Vestroni, F. (2002), "Feasibility of a vibration absorber based on hysterisis", Proc. 3rd World Cong. Str. Contr. Casciati, eds., 421-430.

Larsen, A. (1993), "Vortex-induced response of bridges and control by tuned mass dampers", EURODYN'93, Moan et al., Eds., A.A. Balkema, Rotterdam, 1003-1011.

Markiewicz, M. (1995), "Optimum dynamic characteristics of stockbridge dampers for dead-end spans", $J$. Sound Vib., 188(2), 243-256.

Novak, M. (1969), "Aeroelastic galloping of prismatic bodies”, Eng. Mech. Div., ASCE, 96, 115-130.

Rowbottom, M.D. (1981), "The optimization of mechanical dampers to control self-excited galloping oscillations", J. Sound \& Vib., 75, 559-576.

Sauter, D. and Hagedorn, P. (2002), "On the hysteresis of wire cables in Stockbridge dampers", Int. J. Non-Lin. Mech., 37, 1453-1459.

Strommen, E. and Hjorth-Hansen, E. (2001), "On the use of tuned mass dampers to suppress vortex shedding induced vibrations", Wind Struct., An Int. J., 4(1), 19-30.

\section{Appendix-I}

The nonlinear term of the constitutive relation in Eq. (1) considered in the analyses presented in Section 4 are respectively cubic and Van der Pol's damping as

$$
\begin{aligned}
& f\left(q_{1}, q_{2}, \dot{q}_{1}, \dot{q}_{2}\right)=c_{t n}\left(\dot{q}_{1}-\dot{q}_{2}\right)^{3} \\
& f\left(q_{1}, q_{2}, \dot{q}_{1}, \dot{q}_{2}\right)=c_{t v}\left(q_{1}-q_{2}\right)^{2}\left(\dot{q}_{1}-\dot{q}_{2}\right)
\end{aligned}
$$

consequently the nonlinear vecotr fields of Eq. (2) respectively become, for cubic damping

$$
\begin{aligned}
& f_{1}=0, f_{2}=0 \\
& f_{3}=\frac{1}{2} \frac{\delta^{2} A_{1} A_{3}}{\xi_{s} v} x_{3}^{3}+2 \frac{\mu \xi_{t n}}{\gamma}\left(x_{4}^{3}-x_{3}^{3}\right)+6 \frac{\mu \xi_{t n}}{\gamma}\left(x_{3}^{2} x_{4}-x_{3} x_{4}^{2}\right) \\
& f_{4}=2 \frac{\xi_{t n}}{\gamma}\left(x_{4}^{3}-x_{3}^{3}\right)+6 \frac{\xi_{t n}}{\gamma}\left(x_{3} x_{4}^{2}-x_{3}^{2} x_{4}\right)
\end{aligned}
$$

and for Van der Pol's damping

$$
\begin{aligned}
& f_{1}=0, f_{2}=0 \\
& f_{3}=\frac{1}{2} \frac{\delta^{2} A_{1} A_{3}}{\xi_{s} v} x_{3}^{3}+2 \mu \gamma \xi_{t v}\left(x_{1}^{2}+x_{2}^{2}\right)\left(x_{4}-x_{3}\right)+4 \mu \gamma \xi_{t v} x_{1} x_{2}\left(x_{3}-x_{3}\right) \\
& f_{4}=2 \gamma \xi_{t v}\left(x_{1}^{2}+x_{2}^{2}\right)\left(x_{3}-x_{4}\right)+4 \gamma \xi_{t v} x_{1} x_{2}\left(x_{4}-x_{3}\right)
\end{aligned}
$$

where the non dimensional quantities $\xi_{t n}, \xi_{t v}$ are defined as

$$
\xi_{t n}=\frac{c_{t n} \omega_{t} D^{2}}{2 m_{t}} \quad \xi_{t v}=\frac{c_{t v} \omega_{t} D^{2}}{2 \omega_{s}^{2} m_{t}} \quad \text { with } \quad \xi_{t n}=\frac{\xi_{t v}}{\gamma^{2}}
$$




\section{Appendix - II}

The expressions of the coefficients in Eq. (10) are :

$$
\begin{aligned}
& C_{1}=\mathbf{v}_{2}^{H} \mathbf{L}_{2} \mathbf{u}_{1} \\
& C_{2}=p_{31}+\mathbf{v}_{2}^{H} \mathbf{L}_{2} \mathbf{u}_{2} \\
& C_{3}=\frac{1}{2} \mathbf{v}_{2}^{H} \mathbf{f}_{\mathbf{x x x}}^{0} \mathbf{u}_{1}^{2} \overline{\mathbf{u}}_{1} \\
& C_{4}=2 p_{32} \mathbf{v}_{2}^{H} \mathbf{u}_{2}+\mathbf{v}_{2}^{H} \mathbf{f}_{\mathbf{x x x}}^{0} \mathbf{u}_{1} \overline{\mathbf{u}}_{1} \overline{\mathbf{u}}_{2} \\
& C_{5}=p_{32} \mathbf{v}_{2}^{H} \mathbf{u}_{2}+\frac{1}{2} \mathbf{v}_{2}^{H} \mathbf{f}_{\mathbf{x x x}}^{0} \mathbf{u}_{1}{ }^{2} \overline{\mathbf{u}}_{2} \\
& p_{31}=\mathbf{v}_{1}^{H} \mathbf{L}_{2} \mathbf{u}_{1} \\
& p_{32}=\mathbf{v}_{1}^{H} \mathbf{f}_{\mathbf{x x x}}^{0} \mathbf{u}_{1}^{2} \overline{\mathbf{u}}_{1}
\end{aligned}
$$

In Eqs. (16), $\mathbf{u}_{k}$ and $\mathbf{v}_{k}(k=1,2)$ are the right and the left eigenvectors of the linear operator $\mathbf{L}$ defined by Eq. (2), an overbar denotes the complex conjugate and ()$^{H}$ the transpose conjugate. Moreover, $\mathbf{z}_{111}$ is solution to the following algebraic problem:

$$
\left(\mathrm{L}_{0}-3 i \omega_{0}\right) \mathbf{z}_{111}=\frac{1}{6} \mathbf{f}_{\mathbf{x x x}}^{0} \mathbf{u}_{1}^{3}
$$

Furthermore, the cubic equation obtained by zeroing the right hand side terms of eqns (10a-10c) reads:

$$
\begin{aligned}
& z_{3} \hat{a}^{3}+z_{2} \hat{a}^{2}+z_{1} \hat{a}+z_{0}=0 \\
& z_{0}=64\left(R_{2} I_{1} I_{2}+R_{1} R_{2}+I_{1}^{2}\right) \\
& z_{1}=16 R_{2}\left(R_{2} R_{3}+I_{2} I_{3}\right)+16\left(R_{4}-R_{5}\right)\left(2 R_{1} R_{2}+I_{1} I_{2}\right)+32 I_{1} I_{3}-R_{2} I_{1}\left(I_{5}-I_{4}\right) \\
& z_{2}=4 R_{1}\left(R_{4}-R_{5}\right)^{2}-R_{2} I_{3}\left(I_{5}-I_{4}\right)+8 R_{2} R_{3}\left(R_{4}-R_{5}\right)+4 I_{3}^{2}-4 I_{1}\left(R_{4}-R_{5}\right)\left(I_{5}-I_{4}\right)+I_{2} I_{3}\left(R_{4}-R_{5}\right) \\
& z_{3}=R_{3}\left(R_{4}-R_{5}\right)^{2}-I_{3}\left(R_{4}-R_{5}\right)\left(I_{5}-I_{4}\right)
\end{aligned}
$$

where $\hat{a}=a^{2}$.

$G S$ 